\title{
A Study on Transmuted Half Logistic Distribution: Properties and Application
}

\author{
Adeyinka Femi Samuel, Olapade Akintayo Kehinde* \\ Department of Mathematics, Obafemi Awolowo University, Ile-Ife, Nigeria \\ Email address: \\ fs.adeyinka247@gmail.com (A. F. Samuel), akolapad@oauife.edu.ng (O. A. Kehinde) \\ ${ }^{*}$ Corresponding author
}

\section{To cite this article:}

Adeyinka Femi Samuel, Olapade Akintayo Kehinde. A Study on Transmuted Half Logistic Distribution: Properties and Application. International Journal of Statistical Distributions and Applications. Vol. 5, No. 3, 2019, pp. 54-59. doi: 10.11648/j.ijsd.20190503.12

Received: May 2, 2019; Accepted: June 24, 2019; Published: August 13, 2019

\begin{abstract}
In this article we transmute the half logistic distribution using quadratic rank transmutation map to develop a transmuted half logistic distribution. The quadratic rank transmutation map enables the introduction of extra parameter into its baseline distribution to enhance more flexibility in the analysis of data in various disciplines such as reliability analysis in engineering, survival analysis, medicine, biological sciences, actuarial science, finance and insurance. The mathematical properties such as moments, quantile, mean, median, variance, skewness and kurtosis of this distribution are discussed. The reliability and hazard functions of the transmuted half logistic distribution are obtained. The probability density functions of the minimum and maximum order statistics of the transmuted half logistic distribution are established and the relationships between the probability density functions of the minimum and maximum order statistics of the parent model and the probability density function of the transmuted half logistic distribution are considered. The parameter estimation is done by the method of maximum likelihood estimation. The flexibility of the model in statistical data analysis and its applicability is demonstrated by using it to fit relevant data. The study is concluded by demonstrating that the transmuted half logistic distribution has a better goodness of fit than its parent model. We hope this model will serve as an alternative to the existing ones in the literature in fitting positive real data.
\end{abstract}

Keywords: Half Logistic Distribution, Reliability Function, Hazard Rate Function, Parameter Estimation, Order Statistics, Transmutation

\section{Introduction}

Half logistic probability model has a lot of applications in the analysis of survival data and other disciplines such as economics, biological sciences, insurance and finance. Balakrishnan [1] studied order statistics from the half logistic distribution. Balakrishnan and Puthenpura [2] obtained best unbiased estimates of the location and scale parameter of the distribution. Balakrishnan and Wong [3] obtained approximate maximum likelihood estimates for the location and scale parameters of the half logistic distribution. Olapade [4] presented some theorems that characterized the distribution. Torabi and Bagheri [5] presented an extended generalized half logistic distribution and studied different methods for estimating its parameters based on complete and censored data. The half logistic distribution which was studied by Balakrishnan [1] and Balakrishnan and Puthenpura [2] has the probability density function given by

$$
g(x)=\frac{2 e^{\frac{x-\mu}{\sigma}}}{\sigma\left(1+e^{\frac{x-\mu}{\sigma}}\right)^{2}}, x>0
$$

Transmutation of distributions gained popularity over a decade ago from the work of Shaw et al [6] to the work of, Aryal and Tsokos [7], Aryal and Tsokos [8], Merovci et al [9], Merovci [10], Merovci and Elbata [11], Merovci and Puka [12], Granzoto et al [13], Rahman et al [14], to mention a few.

This work intends to study the transmutation of half logistic distribution given in (1).

\section{Transmuted Half Logistic Distribution}

If a random variable $X$ has half logistic distribution with probability density function (pdf) in (1) with the cumulative distribution function (cdf) given by 


$$
G(x)=\frac{e^{\frac{x-\mu}{\sigma}}-1}{e^{\frac{x-\mu}{\sigma}}+1}, x>0
$$

Where $\mu \leq x$ is the location parameter and $\sigma>0$ is the scale parameter. We assume $\mu=0$ and $\sigma=1$ with no loss of generality.

The corresponding transmuted half logistic distribution, using the quadratic rank transmutation map,

$$
F(x)=(1+x) G(x)-\lambda G^{2}(x),|\lambda| \leq 1
$$

where $|\lambda| \leq 1$ is the transmutation parameter, is given by

$$
F(x)=\frac{\left(e^{x}-1\right)\left(1+2 \lambda+e^{x}\right)}{\left(1+e^{x}\right)^{2}}, x>0,
$$

and the corresponding pdf is obtained by differentiating (4) with respect to $x$ which is given by

$$
f(x)=\frac{2 e^{x}\left\{(1-\lambda) e^{x}+3 \lambda+1\right\}}{\left(1+e^{x}\right)^{3}}, x>0 .
$$

When $\lambda=0$, we have the pdf of the half logistic distribution given in (1) with parameters $\mu=0$ and $\sigma=1$.

Figure 1 and figure 2 illustrate the graphs of pdf and cdf of transmuted half logistic distributions for different values of parameter $\lambda$.

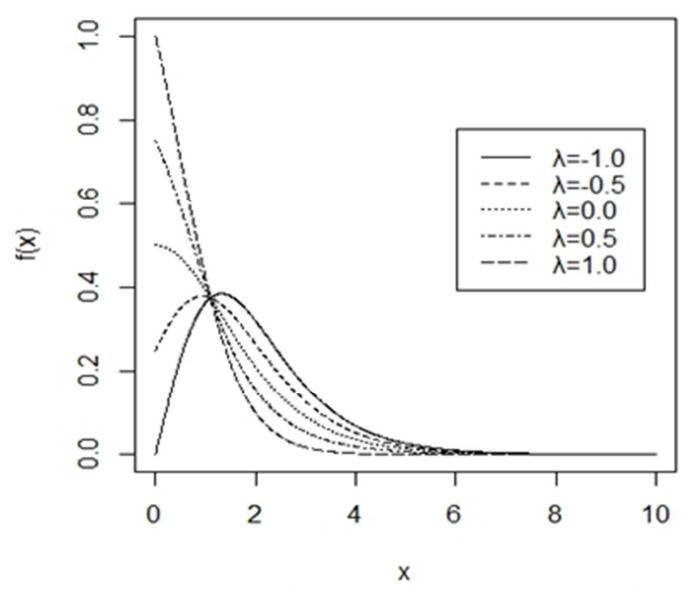

Figure 1. Pdf of transmuted half logistic distribution.

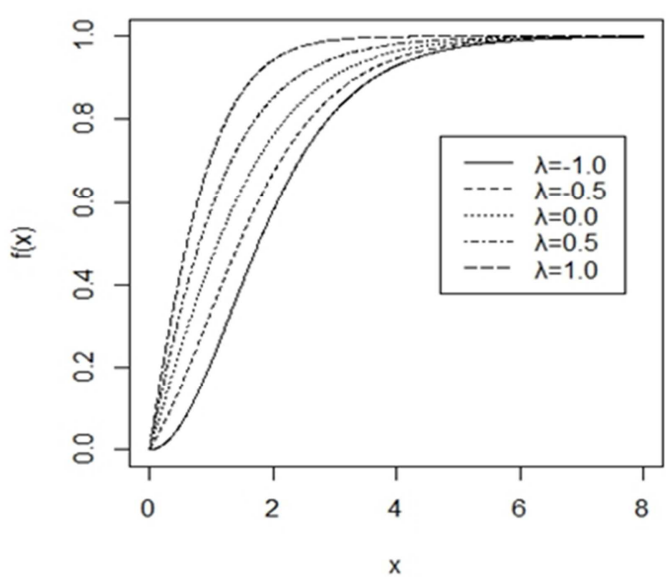

Figure 2. Cdf of transmuted half logistic distribution.

\section{Moments and Quantiles}

The $k^{\text {th }}$ order moment of a transmuted half logistic random variable $\mathrm{x}$, is given by

$$
E\left[X^{k}\right]=2 \int_{0}^{\infty} x^{k} e^{x} \frac{\left\{(1-\lambda) e^{x}+3 \lambda+1\right\}}{\left(1+e^{x}\right)^{3}} d x
$$

The result cannot be obtained explicitly. This is obtained numerically using Maple software. Table 1 shows the moments for various values of parameter $\lambda$.

The $q^{\text {th }}$ quantile $x_{q}$ of the transmuted half logistic distribution can be obtained from (4) as

$$
x_{q}=\ln \left\{\frac{(q-\lambda)+\sqrt{(1+\lambda)^{2}-4 \lambda q}}{(1-q)}\right\}
$$

The median of the transmuted half logistic distribution is obtained when $q=0.5$ in (7) to have

$$
x_{0.5}=\ln \left\{(1-2 \lambda)+2\left(\sqrt{1+\lambda^{2}}\right)\right\}
$$

Table 1. The Moments of the Transmuted Half Logistic Distribution for selected values of parameter $\lambda$.

\begin{tabular}{llllll}
\hline $\boldsymbol{\lambda}$ & $\boldsymbol{E}[\boldsymbol{X}]$ & $\boldsymbol{E}\left[\boldsymbol{X}^{\mathbf{2}}\right]$ & $\boldsymbol{E}\left[\boldsymbol{X}^{\mathbf{3}}\right]$ & $\boldsymbol{E}\left[\boldsymbol{X}^{\mathbf{4}}\right]$ & $\boldsymbol{V a r}[\boldsymbol{X}]$ \\
\hline-1.0 & 2.0000 & 5.5452 & 19.7393 & 86.5481 & 1.5452 \\
-0.75 & 1.8466 & 4.9814 & 17.5090 & 76.2755 & 1.5715 \\
-0.5 & 1.6931 & 4.4175 & 15.2789 & 66.0028 & 1.5510 \\
-0.25 & 1.5397 & 3.8537 & 13.0487 & 55.7302 & 1.4830 \\
0.0 & 1.3863 & 3.2899 & 10.8185 & 45.4576 & 1.3681 \\
0.25 & 1.2329 & 2.7260 & 8.5883 & 35.1849 & 1.2060 \\
0.5 & 1.0794 & 2.1622 & 6.3582 & 24.9123 & 0.9971 \\
0.75 & 0.9260 & 1.5984 & 4.1280 & 14.6397 & 0.7410 \\
1.0 & 0.7726 & 1.0346 & 1.8978 & 4.3671 & 0.4377 \\
\hline
\end{tabular}

\section{The Skewness and Kurtosis of the Transmuted Half Logistic Distribution}

The coefficient of skewness of the Transmuted Half Logistic (I) Distribution is denoted by $\beta_{1}$ such that

$$
\beta_{1}=\frac{\mu_{3}^{2}}{\mu_{2}^{3}}
$$

Where $\mu_{2}=\operatorname{Var}[X]=E\left[X^{2}\right]-(E[X])^{2}$ and $\mu_{3}=$ $E\left[X^{3}\right]-3 E\left[X^{2}\right] E[X]+2(E[X])^{3}$.

The coefficient of Kurtosis of the Transmuted Half Logistic (I) Distribution is denoted by $\beta_{2}$ such that

$$
\beta_{2}=\frac{\mu_{4}}{\mu_{2}^{2}}
$$

Where $\mu_{4}=E\left[X^{4}\right]-4 E\left[X^{3}\right] E[X]+6 E\left[X^{2}\right](E[X])^{2}-3(E[X])^{4}$

The skewness and kurtosis of the transmuted half logistic (I) distribution for selected values of parameter $\lambda$ is shown in Table 2. 
Table 2. The Skewness and Kurtosis of the Transmuted Half Logistic (I) Distribution.

\begin{tabular}{lrcll}
\hline \multicolumn{1}{c}{$\boldsymbol{\lambda}$} & $\boldsymbol{\mu}_{\mathbf{3}}$ & $\boldsymbol{\mu}_{\mathbf{4}}$ & Skweness $\left(\boldsymbol{\beta}_{\mathbf{1}}\right)$ & $\operatorname{Kurtosis}\left(\boldsymbol{\beta}_{\mathbf{2}}\right)$ \\
\hline-1.0 & 2.4680 & 13.7164 & 1.65 & 5.74 \\
-0.75 & 2.5066 & 13.9815 & 1.62 & 5.66 \\
-0.5 & 2.5066 & 13.8549 & 1.74 & 5.76 \\
-0.25 & 2.5483 & 13.3208 & 1.99 & 6.06 \\
0 & 2.4646 & 12.3223 & 2.37 & 6.58 \\
0.25 & 2.2538 & 10.7611 & 2.90 & 7.40 \\
0.5 & 1.8718 & 8.5029 & 3.53 & 8.55 \\
0.75 & 1.2757 & 5.3673 & 3.40 & 9.76 \\
1.0 & 0.4222 & 1.1386 & 2.13 & 5.94 \\
\hline
\end{tabular}

\section{Random Number Generation and Parameter Estimation}

Using the method of inversion we can generate random numbers from the transmuted half logistic distribution as

$$
\frac{\left(e^{x}-1\right)\left(1+2 \lambda+e^{x}\right)}{\left(1+e^{x}\right)^{2}}=u
$$

where $u \sim U(0,1)$. After simplifying (9) we have

$$
x=\ln \left\{\frac{(u-\lambda)+\sqrt{(1+\lambda)^{2}-4 \lambda u}}{(1-u)}\right\}
$$

which can be used to generate random numbers when the parameter $\lambda$ is known.

The maximum likelihood estimates of the parameters $\lambda, \mu$ and $\sigma$ that are inherent within the transmuted half logistic distribution function are given by the following:

Let $X_{1}, X_{2}, \ldots X_{n}$ be a sample of size $\mathrm{n}$ from a transmuted half logistic distribution. The likelihood function is given by

$$
\begin{gathered}
L=2^{n} \sigma^{-n} e^{\sum_{i=n}^{n}\left(\frac{x_{i}-\mu}{\sigma}\right)} \prod_{i=1}^{n}\left\{(1-\lambda) e^{\sum_{i=n}^{n}\left(\frac{x_{i}-\mu}{\sigma}\right)}+3 \lambda+1\right\} / \\
\prod_{i=1}^{n}\left(1+e^{\sum_{i=n}^{n}\left(\frac{x_{i}-\mu}{\sigma}\right)}\right)^{3}
\end{gathered}
$$

Hence the log-likelihood function becomes

$$
\begin{gathered}
\ln L=n \ln 2-n \ln \sigma+\sum_{i=n}^{n}\left(\frac{x_{i}-\mu}{\sigma}\right)+\sum_{i=1}^{n} \ln \left\{(1-\lambda) e^{\sum_{i=n}^{n}\left(\frac{x_{i}-\mu}{\sigma}\right)}+3 \lambda+1\right\} \\
-3 \sum_{i=1}^{n} \ln \left(1+e^{\sum_{i=n}^{n}\left(\frac{x_{i}-\mu}{\sigma}\right)}\right)
\end{gathered}
$$

By differentiating (12) with respect to parameters $\mu, \sigma$ and $\lambda$ and equating the result to zero we have

$$
\begin{gathered}
\frac{\partial \ln L}{\partial \lambda}=\sum_{i=1}^{n} \frac{3-e^{\left(\frac{x_{i}-\mu}{\sigma}\right)}}{\left[(1-\lambda) e^{\left(\frac{x_{i}-\mu}{\sigma}\right)}+3 \lambda+1\right]}=0 \frac{\partial \ln L}{\partial \mu}=-\sigma^{-1}\left\{n+(1-\lambda) \sum_{i=1}^{n} \frac{e^{\left(\frac{x_{i}-\mu}{\sigma}\right)}}{\left[(1-\lambda) e^{\left(\frac{x_{i}-\mu}{\sigma}\right)}+3 \lambda+1\right]}-3 \sum_{i=1}^{n} \frac{e^{\left(\frac{x_{i}-\mu}{\sigma}\right)}}{\left[1+e^{\left(\frac{x_{i}-\mu}{\sigma}\right)}\right]}\right\}=0 \frac{\partial \ln L}{\partial \sigma} \\
=-\sigma^{-2}\left\{n \sigma+\sum_{i=1}^{n}\left(x_{i}-\mu\right)-(1-\lambda) \sum_{i=1}^{n} \frac{\left(x_{i}-\mu\right) e^{\left(\frac{x_{i}-\mu}{\sigma}\right)}}{\left[(1-\lambda) e^{\left(\frac{x_{i}-\mu}{\sigma}\right)}+3 \lambda+1\right]}+3 \sum_{i=1}^{n} \frac{\left(x_{i}-\mu\right) e^{\left(\frac{x_{i}-\mu}{\sigma}\right)}}{\left[1+e^{\left(\frac{x_{i}-\mu}{\sigma}\right)}\right]}\right\}=0
\end{gathered}
$$

The maximum likelihood estimator $\hat{\theta}=(\hat{\mu}, \hat{\sigma}, \hat{\lambda})^{\prime}$ of parameters $\theta=(\mu, \sigma, \lambda)^{\prime}$ can be obtained solving this nonlinear system of equations. It is usually more convenient to use non-linear optimization algorithms such as quasi-Newton algorithm to numerically maximize the log-likelihood function in (12).

\section{Reliability Analysis}

The survival function, also known as the reliability function in engineering, is the characteristic of the explanatory variable that maps a set of events, usually associated with mortality or failure of some system unto time. It is the probability that a system will survive beyond a specified time.

The transmuted half logistic distribution can be a useful model to characterize failure time of a given system because of the analytical structure. The reliability function $R(t)$, which is the probability of an item not failing prior to some time $t$, is defined by $R(t)=1-F(t)$. The reliability function of the half transmuted logistic distribution is given by

$$
R(t)=\frac{2\left\{\left(1+e^{t}\right)-\lambda\left(e^{t}-1\right)\right\}}{\left(1+e^{t}\right)^{2}} .
$$

Figure 3 and figure 4 illustrate the reliability behaviour of transmuted half logistic distribution as the value of parameter $\lambda$ varies from -1 to 1 .
The other characteristics of interest of a random variable is the hazard rate function also known as instantaneous failure rate defined by

$$
h(t)=\frac{f(t)}{1-F(t)}
$$

which is an important quantity characterizing life phenomenon. It can be loosely interpreted as the conditional probability of failure, given it has survived to the time $t$. The hazard rate function of a transmuted half logistic distribution is given by

$$
h(t)=\frac{e^{t}\left\{(1-\lambda) e^{t}+(3 \lambda-1)\right\}}{\left(1+e^{t}\right)\left[\left(1+e^{t}\right)-\lambda\left(e^{t}-1\right)\right]} .
$$

Figure 2(ii) illustrates the behavior of hazard rate function of a transmuted $\lambda \log$-logistic distribution for the selected values of parameter $\lambda$.

The cumulative hazard function of a transmuted half logistic distribution is given by

$$
H(t)=\int_{0}^{t} h(x) d x=\ln \left\{\frac{\left(1+e^{t}\right)^{2}}{2\left[((1+\lambda))+(1-\lambda) e^{t}\right]}\right\} .
$$

It is observed that

i. $H(t)$ is a non-decreasing for all $t \geq 0$

ii. $H(0)=0$

iii. $\lim _{t \rightarrow \infty} H(t)=\infty$. 


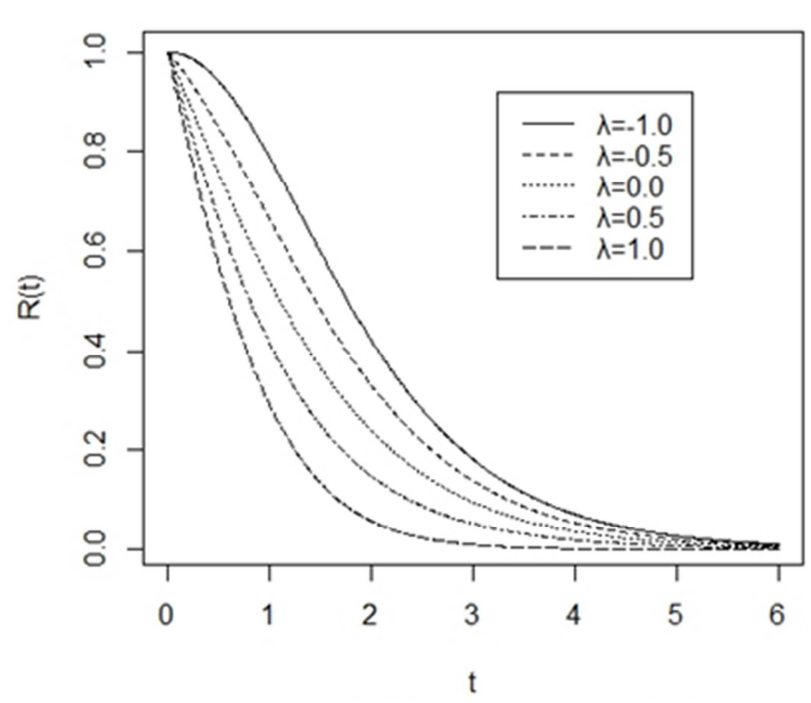

Figure 3. The reliability function of half logistic distribution.

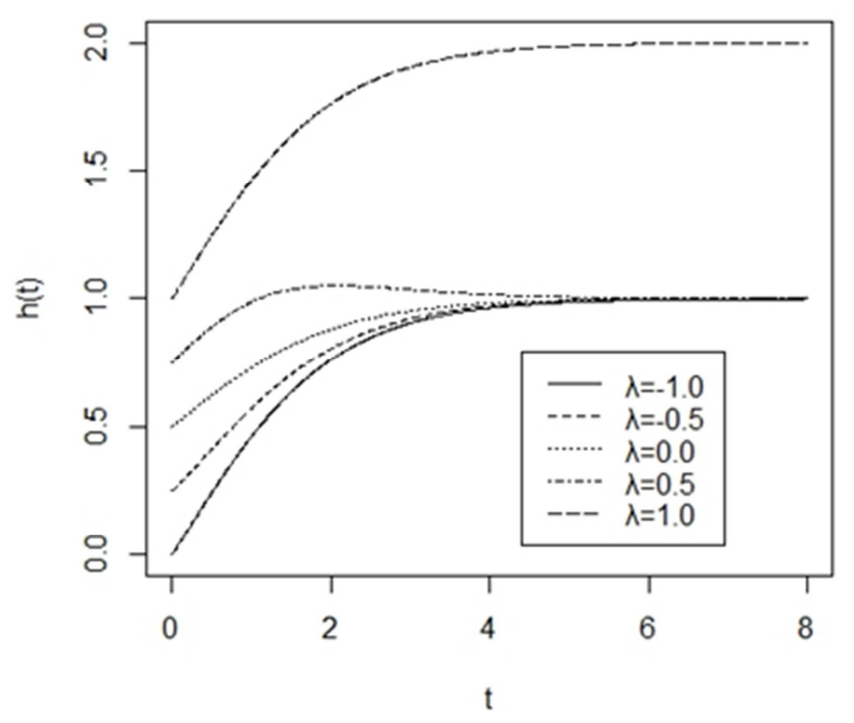

Figure 4. The hazard function of transmuted half logistic distribution.

Given that a unit is of age $t$, the remaining time after time $t$ is random. The expected value of this random residual life is called the mean residual life(MRL) at time t. The mean residual life (MRL) at a given time $t$ measures the expected remaining life time of an individual of age $t$. It is given by

$$
\begin{aligned}
& m(t)=E(T-t / T \geq t) \\
& =\frac{1}{R(t)} \int_{t}^{\infty} R(u) d u
\end{aligned}
$$

Note that $\mathrm{m}(0)$ is the mean time to failure. The MRL can be expressed in terms of the cumulative hazard rate function as

$$
\begin{aligned}
& m(t)=\int_{0}^{\infty} e^{[H(t)-H(t+x)]} d x . \\
& f_{X_{(r)}}(x)=\frac{2 e^{x}\left\{(1-\lambda) e^{x}+3 \lambda+1\right\}\left\{\left(e^{x}-1\right)\left(1+2 \lambda+e^{x}\right)\right\}^{r-1}\left\{\left(1+e^{x}\right)^{2}-\left(e^{x}-1\right)\left(1+2 \lambda+e^{x}\right)\right\}^{n-r}}{B(r, n-r+1)\left(1+e^{x}\right)^{2 n+1}}
\end{aligned}
$$

The mean residual life of the transmuted half logistic distribution is obtained by substituting $R(t)$ into (13) to have

$$
m(t)=\frac{\left(1+e^{t}\right)^{2}}{\left(1+e^{t}\right)-\lambda\left(e^{t}-1\right)} \int_{t}^{\infty} \frac{\left(1+e^{u}\right)-\lambda\left(e^{u}-1\right)}{\left(1+e^{u}\right)^{2}} d u
$$

The integral in (14) gives $2\left(1+e^{t}\right)^{-1}-(1+$ d) $\ln \left[e^{t}\left(1+e^{t}\right)^{-1}\right]$.

By substituting this back into (14) it becomes

$$
m(t)=\frac{2-(1+\lambda)\left(1+e^{t}\right) \ln \left[e^{t}\left(1+e^{t}\right)^{-1}\right]}{1-\lambda\left(e^{t}-1\right)\left(1+e^{t}\right)^{-1}}
$$

\section{Order Statistics}

We know that if $X_{(1)} \leq X_{(2)} \leq \cdots \leq X_{(n)}$ denotes the order statistics of a random sample $X_{1}, X_{2} \ldots X_{n}$ from a continuous population with $\operatorname{cdf} F_{X}(x)$ and $\operatorname{pdf} f_{X}(x)$, David 1970 gave the probability density function of $X_{(r)}$ as

$$
f_{X_{(r)}}(x)=\frac{1}{B(r, n-r+1)}[F(x)]^{r-1}[1-F(x)]^{n-r} f(x)
$$

where $r=1,2, \ldots n$.

We have from (1) and (2) the pdf of the $r^{\text {th }}$ order half logistic random variable $X_{(r)}$ given by

$$
g_{X_{(r)}}(x)=\frac{2^{n-r+1} e^{x}\left(e^{x}-1\right)^{r-1}}{B(r, n-r+1)\left(1+e^{x}\right)^{n+1}}
$$

Therefore the pdf of the $n t h$ order half logistic statistic $X_{(n)}$ is obtained by taking $r=n$ in (17) and it is given by

$$
g_{X_{(n)}}(x)=\frac{2 n e^{x}\left(e^{x}-1\right)^{n-1}}{\left(1+e^{x}\right)^{n+1}}
$$

and the pdf of the $1^{\text {st }}$ order half logistic statistic $X_{(1)}$ is obtained by taking $r=1$ in (17) and it is given by

$$
g_{X_{(1)}}(x)=\frac{2^{n} n e^{x}}{\left(1+e^{x}\right)^{n+1}}
$$

Note that in a particular case of $n=2,(18)$ yields

$$
g_{X_{(n: 2)}}(x)=\frac{2^{2} e^{x}\left(e^{x}-1\right)}{\left(1+e^{x}\right)^{3}}
$$

and (19) yields

$$
g_{X_{(1: 2)}}(x)=\frac{2^{3} e^{x}}{\left(1+e^{x}\right)^{3}}
$$

It can be observed that $\min \left(X_{1}, X_{2}\right)$ and $\max \left(X_{1}, X_{2}\right)$ are special cases of (5) for $\lambda=1$ and $\lambda=-1$ respectively.

We have from (4) and (5) the pdf of the $r^{\text {th }}$ order statistics for the transmuted half logistic distribution given by 

by

Therefore the pdf of the largest order statistic $X_{(n)}$ is given

$$
f_{X_{(n)}}(x)=\frac{2 n e^{x}\left\{(1-\lambda) e^{x}+3 \lambda+1\right\}\left\{\left(e^{x}-1\right)\left(1+2 \lambda+e^{x}\right)\right\}^{n-1}}{\left(1+e^{x}\right)^{2 n+1}}
$$

and the pdf of the smallest order statistic $X_{(1)}$ is given by

$$
f_{X_{(1)}}(x)=\frac{2 n e^{x}\left\{(1-\lambda) e^{x}+3 \lambda+1\right\}\left\{\left(1+e^{x}\right)^{2}-\left(e^{x}-1\right)\left(1+2 \lambda+e^{x}\right)\right\}^{n-1}}{\left(1+e^{x}\right)^{2 n+1}}
$$

\section{Application}

The data represents the survival time of 72 Guinea Pigs infected with virulent tubercle bacilli. The data set is obtained from the work of Usman, Haq and Talib (2017) [15].

The data are as follows:

$0.1,0.33,0.44,0.56,0.59,0.72,0.74,0.77,0.92,0.93$, $0.96,1.00,1.00,1.02,1.05,1.07,0.7,0.08,1.08,1.08,1.09$, $1.12,1.13,1.15,1.16,1.20,1.21,1.22,1.22,1.24,1.3,1.34$, $1.36,1.39,1.44,1.46,1.53,1.59,1.60,1.63,1.63,1.68,1.71$, $1.72,1.76,1.83,1.95,1.96,1.97,2.02,2.13,2.15,2.16,2.22$, $2.30,2.31,2.40,2.45,2.51,2.53,2.54,2.54,2.78,2.93,3.27$, $3.42,3.47,3.61,4.02,4.32,4.58,5.55$.

A quasi Newton algorithm was implemented in R package and the performances of the models are shown in Table 3. Akaike Information criterion (AIC), Corrected Akaike Information criterion (AICC) and Bayesian Information criterion (BIC) were respectively used to compare the performance of type I generalized half logistic distribution (THL) to its parent model (HL) in (2).

$$
\begin{gathered}
A I C=2 k-2 L L \\
A I C C=A I C+\frac{2 k(k+1)}{n-k-1}
\end{gathered}
$$

And

$$
B I C=2 \log (n)-2 L L
$$

Where $\mathrm{k}$ is the number of parameters in the model, $\mathrm{n}$ is the sample size and LL is the maximized value of log likelihood function.

Table 3. Performance of the models.

\begin{tabular}{llllll}
\hline Model & Estimates & -LL & AIC & AICC & BIC \\
\hline \multirow{2}{*}{ THL } & $\hat{\mu}=1.091$ & & & & \\
& $\hat{\sigma}=0.0062$ & 151.321 & 308.642 & 308.995 & 306.357 \\
\multirow{2}{*}{ HL } & $\hat{\lambda}=0.9361$ & & & & \\
& $\hat{\mu}=1.2152$ & \multirow{2}{*}{157.341} & 314.682 & 315.035 & 316.539 \\
\hline
\end{tabular}

It is observed that the transmuted half logistic distribution (THL) performs better than its parent model (HL) in (1).

\section{Conclusion}

In this article, we have introduced a new generalization of the half logistic distribution called transmuted half logistic distribution. The distribution which is generalized by using the quadratic rank transmutation map. Some mathematical properties along with estimation issues are addressed. The hazard rate function and reliability behaviour of the transmuted half logistic distribution shows that the subject distribution can be used to model positive real life data. We hope that this study will serve as a reference and help to advance future research in this field and other related disciplines.

\section{References}

[1] Balakrishnan N. (1985). Order Statistics from the half logistic distribution. Journal of Statistical Computation and Simulation. 20 (4): 287-309.

[2] Balakrishnan N., and Puthenpura, S. (1986). Best linear unbiased estimators of location and scale parameters of the half logistic distribution. Journal of Statistical Computation and Simulation., 25, 193-204.

[3] Balakrishnan N., Wong K. H. T (1991). Approximate MLEs for the Location and Scale Parameters of Half-Logistic Distribution with Type-II Right-Censoring. IEE Transactions on Reliability. 40 (2), 140-145.

[4] Olapade, A. K. (2003). On Characterizations of the Half Logistic Distribution. InterStat, Feburary Issue, 2, http://interstat.stat.vt.edu/InterStat/ARTICLES/2003articles/F 06002.pdf

[5] Torabi, H, and Bagheri, F. L. (2010). Estimation of Parameters for an Extended Generalized Half Logistic Distribution Based on Complete Censored Data. JIRSS, 9 (2), 171-195.

[6] Shaw, W. T, and Buckley, I. R. (2009). Alchemy of Probability Distributions: Beyond Gram-Charlier and Cornish -Fisher Expansions, and Skewed- kurtotic Normal Distribution from a Rank Transmutation Map. arxivpreprint arxiv: 0901.0434.

[7] Aryal, G. R, and Tsokos, C. P. (2009). On the transmuted extreme value distribution with application. Nonlinear Analysis: Theory, Methods and Application. 71 (12), el401el407.

[8] Aryal, G. R, and Tsokos, C. P. (2011). Transmuted Weilbull distribution: A generalization of Weilbull probability distribution. European Journal of Pure and Applied Mathematics. 4 (2), 89-102.

[9] Merovci, F., Alizadeh, M., and Hamedani, G. (2016). Another Generalized Transmuted Family of Distributions: Properties and Applications. Austrian Journal of Statistics. 45, 71-93.

[10] Merovci, F. (2014). Transmuted Generalized Rayleigh Distribution. Journal of Statistics Applications and Probability. 3 (1), 9-20.

[11] Merovci, F., Elbatal, I. (2014). Transmuted Lindley-geometric Distribution and its Applications. Journal of Statistics Applications and Probability. 3 (1), 77-91.

[12] Merovci, F., Puka, L. (2014). Transmuted Pareto Distribution. Probstat. 7, 1-11. 
[13] Rahman M. M, Al-Zahrani B, Shahbaz M. Q (2018). A general transmuted family of distributions. Pak J Stat Oper Res 14:451-469.

[14] Granzoto, D. C. T., Louzada, F., and Balakrishnan, N. (2017). Cubic rank transmuted distributions: Inferential issues and applications. Journal of statistical Computation and
Simulation. 87: 2760-2778, doi: $10-$ 1080/00949655.2017.1344239.

[15] Usman, R. M, Haq, M. A and Talib, J (2017). Kumaraswamy Half-Logistic Distribution: Properties and Applications. Journal of Statistics Applications and Probability. No 3, 597609 . 